\title{
Agriculture Wireless Temperature and Humidity Sensor Network Based on ZigBee Technology
}

\author{
Xi Wang ${ }^{1}$ and Hui Gao ${ }^{2}$ \\ ${ }^{1}$ Heilongjiang Bayi Agricultural Reclamation University, Daqing 163319, China \\ ${ }^{2}$ Lanzhou Jiaotong University, Lanzhou 730070
}

\begin{abstract}
Combining with agricultural production practice, we propose the agriculture wireless and humidity sensor network design, which is based on ZigBee technology. And we use the chip based on the CC2530 ZigBee protocol as the sensor nodes and coordinator nodes for data collection, transmission and display, aiming to realize the agricultural production automation and precision agriculture.
\end{abstract}

Keywords: agricultural production, temperature and humidity, wireless network, sensor.

\section{Introduction}

At present, many aspects of production and life need extracting and processing temperature and humidity information of the surrounding environment. In the past, technology was to collect temperature and humidity information by the temperature and humidity sensors, and to transmit the data to the monitoring center by the RS-485 bus or field bus again, so you need to lay a lot of cables to collect and process temperature and humidity information. Traditional agriculture mainly uses isolated, no communication ability of mechanical equipment, and mainly relies on the person to monitor crop growth status. However, if ZigBee wireless sensor network technology is used, the agriculture will gradually shift to information and software-centric mode of production, and use more automation, networking, intelligent, and remote control equipment to farming. Sensors may collect the information such as soil moisture, nitrogen concentration, $\mathrm{pH}$, precipitation, temperature, air humidity, air pressure and so on. The above-mentioned information and the locations of the collected information are passed to the central control equipment for decision-making and reference through the ZigBee network, so we can identify problems early and accurately for helping to maintain and increase crop yield. In many data-oriented wireless network transmissions, low cost and complexity wireless network are widely used.

\section{$2 \quad$ ZigBee Technical Features}

ZigBee technology is a short distance, low complexity, low power, low data rate, and low-cost, two-way wireless communication technology, employed mainly in the field 
of automatic control and remote control, can be embedded in a variety of devices to achieve their automation [1]. For the various existing wireless communication technology, ZigBee technology will be the lowest power consumption and cost technology.

Transfer rate of ZigBee data is low, ranging from $10 \mathrm{~KB} / \mathrm{s}$ to $250 \mathrm{~KB} / \mathrm{s}$ and focusing on low-transmission applications. Under low power standby mode, the two ordinary No.5 batteries can last 6 to 24 months. ZigBee data transfer rate is low, and its protocol is simple, so it greatly reduces the cost. Network capacity is large to accommodate 65,000 devices. Delay time is short, usually in the $15 \mathrm{~ms} \sim 30 \mathrm{~ms}$. ZigBee provides data integrity checking and authentication capabilities, using AES128 encryption algorithm. Flexible working band, the use of frequency band $2.4 \mathrm{GHz}$, and frequency bands are unlicensed. Such good points as the reliable transmission and the use of collision avoidance strategies are included while the business that requires a fixed bandwidth is reserved specific time slots.

\section{Overall System Design Concept}

Based on ZigBee wireless technology, the temperature and humidity sensor network is composed of three parts: the transmitter, receiver and display system. Transmitter is constituted by a number of terminal nodes; each node consists of a temperature and humidity sensor and a ZigBee wireless RF module. Greenhouse temperature and humidity sensors collect information on temperature and humidity, then temperature and humidity data is transmitted to the ZigBee wireless RF modules. Correction of the temperature and humidity data is processed via a chip embedded in ZigBee wireless RF (radio frequency) module, and the data revised will be sent to the receiver through ZigBee wireless network. Receiver consists of a ZigBee RF module and an RS232 serial port module. Receiver module that is a network coordinator establishes a star structure of the network. The data of each node is received through ZigBee network and transmitted to the display system via RS232. This is the temperature and humidity collection and delivery process of a sending node of the system.

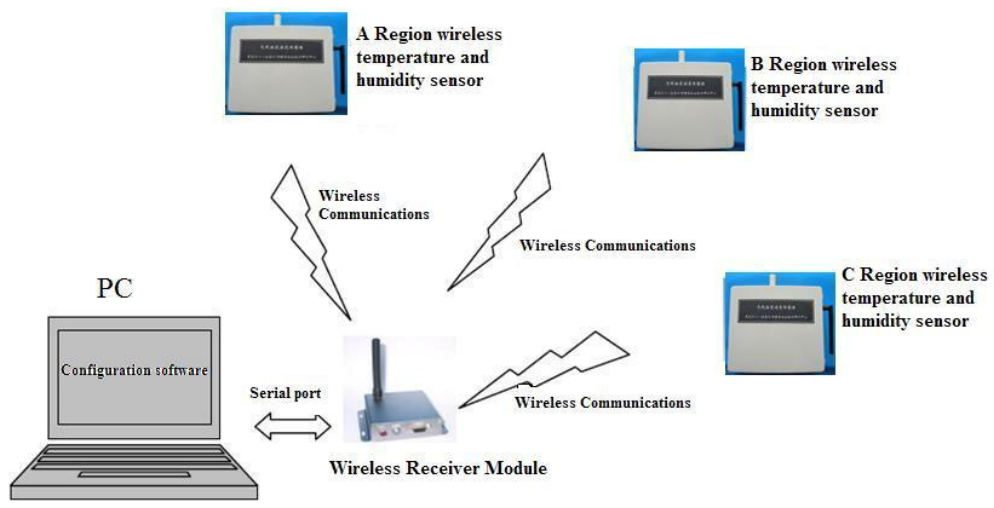

Fig. 1. Overall system simulating scheme 


\section{System Hardware Design}

CC2530 meets low-cost and low power requirements of the $2.4 \mathrm{GHz}$ ISM band based on ZigBee. It includes a high-performance $2.4 \mathrm{GHz}$ DSSS (direct sequence spread spectrum) RF transceiver core and a 8051 controller. ZigBee RF front end, memory and microcontroller are integrated on a single chip. It has $128 \mathrm{kB}$ programmable flash memory and $8 \mathrm{kB}$ RAM, including ADC, timers, $32 \mathrm{kHz}$ crystal sleep mode timer, power-on reset circuit, power-down monitor circuit and 20 programmable I/O pins, enabling the node miniaturization [2]. CC2530 wireless single chip is characterized by very low power, standby current consumption of only $0.2 \mu \mathrm{A}$. Under the run of 32 $\mathrm{kHz}$ crystal clock, the current consumption is less than $1 \mu \mathrm{A}$.

The temperature and humidity sensor SHT11 integrates several circuits into a single chip, such as temperature and humidity detection, signal amplification conditioning, A/D conversion and digital communications interface. Humidity measuring range is $0 \sim 100 \% \mathrm{RH}$, temperature measurement range is $-40^{\circ} \mathrm{C} \sim$ $+123.8^{\circ} \mathrm{C}$, humidity measuring accuracy is $\pm 3.0 \% \mathrm{RH}$, temperature measuring accuracy is $\pm 0.4^{\circ} \mathrm{C}$, and the response time is less than $4 \mathrm{~s}$. For digital interfaces, SHT11 provides two-wire digital serial interface SCK and DATA; SCK is the serial clock line for communication between the microprocessors to achieve synchronization. DATA serve as the serial data line, and makes the data transmission between the microprocessors. The chip interface is simple, good at high transmission reliability, and measurement precision can be adjusted by programming. After the measurement and communication, low-power mode is automatically transferred.

\subsection{The Hardware Design of the Transmitting Node}

Transmitting node is the basic unit of the network composed of digital temperature and humidity sensor SHT11 module, CC2530 processor module, antenna module, power supply module. It is responsible for acquiring temperature and humidity data and data preprocessing, and they will be transmitted to ZigBee receiving end. Temperature and humidity sensor module is responsible for collecting and detecting temperature and humidity data of region. Processor module makes analog-digital conversion for the collected data signal, and then processes. The processed data is issued by the antenna [3]. The power module mainly supplies power for the processor. Transmitting hardware framework is shown in Fig. 2.

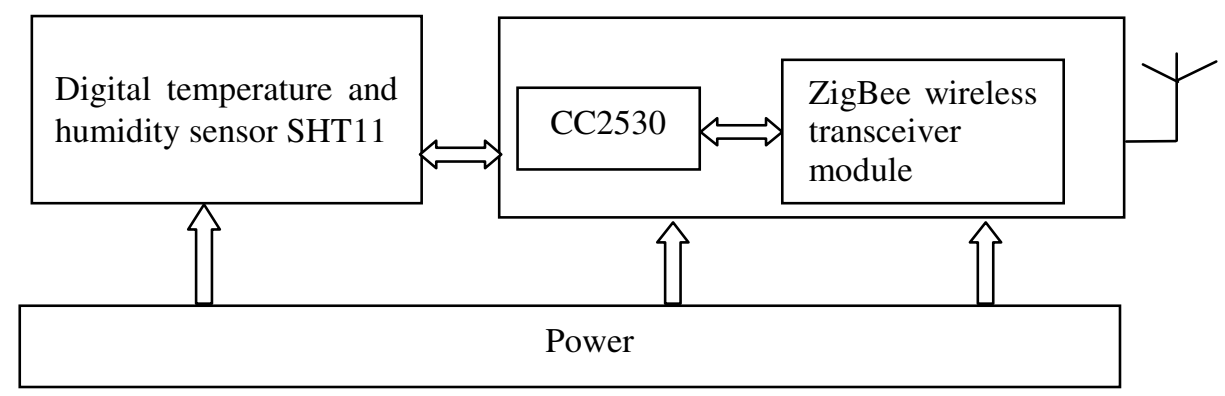

Fig. 2. Transmitting hardware framework 


\subsection{The Hardware Design of the Receiving Node}

Receiver node is composed of a power supply module, a key module, a serial module, a LCD module, LED lights, a CC2530 processor module and an antenna module. The wireless temperature and humidity sensor network is not an independent communication network, which needs transmitting the monitoring temperature and humidity data to the host computer and displaying them. LED indicator lights are used to display the receiving node network state information (e.g. whether the network is built successfully); LCD module is an interface of the user and sensor network to display the function menu, and the user selects them by pressing buttons. CC2530 is a data-receiving mode. When data is received, RF receiving signals are amplified by the low noise amplifier before they are overturned into the frequency mixer. By mixing frequency, IF (intermediate frequency) signal is produced. In IF processing stage, before the signal into the demodulator is amplified and filtered, the data of demodulation is put into the shift register, and then into RFBUF. After the temperature and humidity data in RFBUF are removed by MCU, they will be put into the data buffer register SBUF of UART, and be transmitted to the host computer through RS232 serial module

\section{System Software Design}

The system using development environment is IAR7.51A while the using protocol stack is Z-STACK of TI. The system connecting the receiving end to PC via the serial

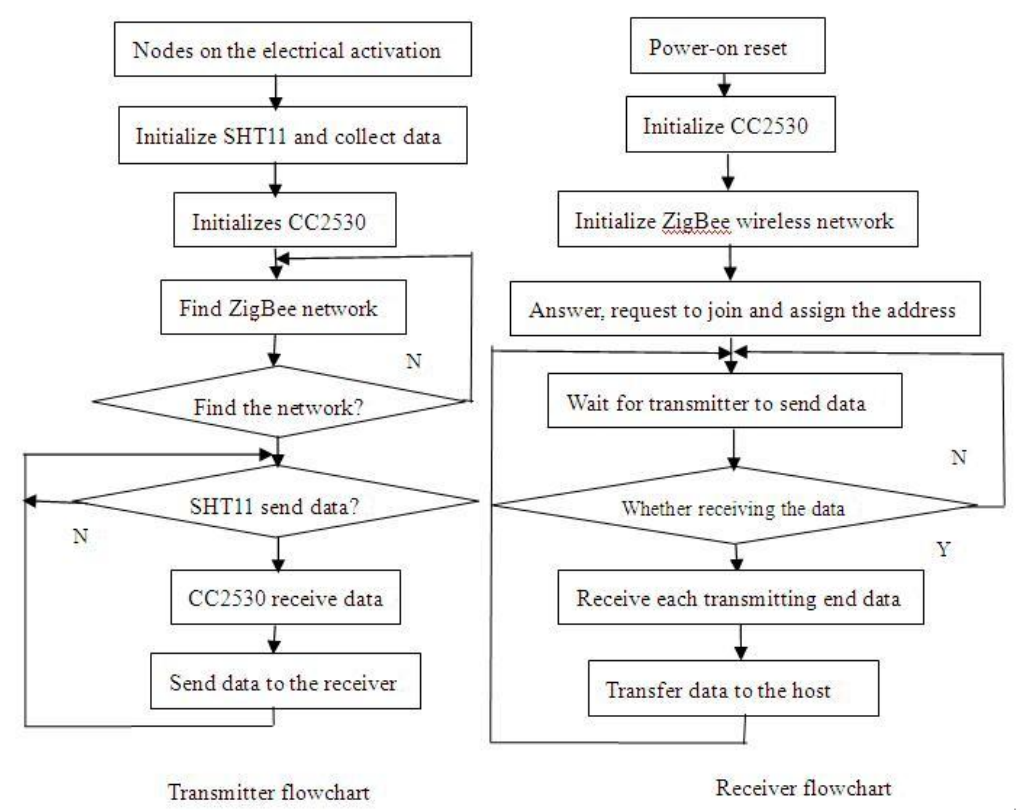

Fig. 3. Transmitter and receiver software flowchart 
port RS232 must know the network address of each sensor node, requiring that the network address is sent to the receiver after each sensor device joins the network. The receiver receiving the network address of the sensor establishes address table to store so that the user collects data of each sensor according to address table when collecting data [4]. Transmitter and receiver software flowchart is shown in Fig. 3.

\section{Construction of Wireless Sensor Networks Based on ZigBee}

The FFD (full functional device) must lead as the network coordinator to establish a network, then the other FFD or RFD join the network, but the RFD (reduce functional device)can only link to FFD. According to the device function in the network, programs are designed beforehand for the device. The coordinator function is to search by scanning and scanning 16 channels set the applicable optimum channel to start a network. The device can form a network (coordinator) in the free channel, or connect to an existed network. Router function is to search by scanning to find an active channel and connect it, then allow connecting other installations. The function of terminal devices is always trying to connect to an existed network. Those terminal devices search other devices that can provide complete search service in the network allowing any network device to initialize the service search [5], and can bind other devices that will provide full service, offering commands and control features for the specified set of conforming devices.

Central control center is connected to a number of the sending end through the network connection. Wireless information transmission between the receiver and transmitter is achieved via Zig Bee technology. The sender is responsible for detecting and processing data and sends it to the receiver. The control center gets the collected relevant information through the network. Multiple senders are distributed in the sensor network, so polling scan is put to the upload data from different ID sink nodes and data of sink nodes can be orderly, completely transmitted after being treated through MCU.

\section{Conclusions}

This paper proposes a wireless temperature and humidity sensor network system based on ZigBee technology, and networking ways are of flexibility, adaptability. Through the practical application in the laboratories and its surrounding offices, this system is proved to be very practical. In practical applications, terminal device number is determined by achieving goal demands. The system can be applied to agricultural production, as well as in more areas of production and life, solving monitor environmental temperature and humidity problems in the areas of high cost and inconvenience arrival of wired network cabling, abominable environment. ZigBee networking ways typical of simple and low power consumption make the temperature and humidity information in real-time delivery reduce system costs, save energy, and conserve resources, and its low cost for the promotion in industry provides convenience. With ZigBee chips at lower prices, the new temperature and humidity monitoring system will also have a broad application prospect. 


\section{References}

1. Zhai, L., Liu, S., Hu, Y.: ZigBee Technology and Application, pp. 313-347. Beijing Aeronautics and Astronautics Press (2007)

2. Chipcon semiconductor. CC2530 Data Sheet 1.02, http: / /www. chipcon. com

3. Liu, Y., Wang, C., Yu, C.: Research on ZigBee wireless Sensors Network Based on ModBus Protocol. Information Technology and Applications, 487-491 (May 2009)

4. Chipcon semiconductor. Chipcon IAR User Manual, http: / /www. chipcon. com

5. Song, J.: Wireless sensor network node design and implementation. Jilin University School of Instrument Science and Electrical Engineering 